\title{
EXISTENCE PROOF FOR A FIELD OF EXTREMALS TANGENT
}

\section{TO A GIVEN CURVE*}

BY

\author{
OSKAR BOLZA
}

In a recent paper, $\dagger$ Professor BuIss has given sufficient conditions for a minimum of the integral

$$
J=\int_{t_{0}}^{t_{1}} F\left(x, y, x^{\prime}, y^{\prime}\right) d t
$$

with respect to one-sided variations. His proof is based upon the construction of a field of extremals tangent to a given curve. He establishes the existence of such a field first for the special case where all curves considered are representable in the form $y=f(x)$, and then reduces the general case of parameter representation to the former by a point-transformation of the plane.

The object of the following note is to give a direct proof for the existence of these fields which play an important part also in other investigations of the calculus of variations. $\ddagger$

\section{§1. The set of extremals tangent to a given curve.}

The terminology and assumptions concerning the function $F$ being the same as in $\S 24$ of my Lectures on the Calculus of Variations, we consider a curve of class $C^{\prime \prime}$

$$
\tilde{\mathfrak{C}}: \quad x=\tilde{x}(a), \quad y=\tilde{y}(a), \quad A_{1} \leqq a \leqq A_{2},
$$

without multiple points, which lies in the interior of the region of the $x, y$ plane in which the function $F$ is supposed to be of class $C^{\prime \prime \prime}$ for every $\left(x^{\prime}, y^{\prime}\right) \neq(0,0)$, and satisfies the inequality

* Presented to the Society March 30, 1907. Received for publication February 27, 1907.

†Transactions of the American Mathematical Society, vol. 5 (1904), p. 477.

† Compare Lindebera, Mathematische Annalen, vol. 59 (1904), p. 321. 


$$
F_{1}\left[\tilde{x}(a), \tilde{y}(a), \tilde{x}^{\prime}(a), \tilde{y}^{\prime}(a)\right]>0 \text { in }\left(A_{1} A_{2}\right),
$$

where $\tilde{x}^{\prime}=d \tilde{x} / d a, \tilde{y}^{\prime}=d \tilde{y} / d a$.

For simplicity, we suppose that the parameter $a$ is the arc of the curve $\tilde{\mathbb{E}}$ measured from some fixed initial point.

Under these conditions it follows from the general existence theorems* for differential equations applied to the differential equation of the extremals $\dagger$ for the integral (1), that through every point $P(a)$ of the curve $\widetilde{\mathbb{E}}$ one and but one extremal $\mathbb{E}_{a}$ can be drawn which is tangent to $\widetilde{\mathbb{E}}$ at $P$ in such a manner that the positive tangents of the two curves coincide. For the parameter $t$ on the extremal $\mathbb{E}_{a}$ we may choose the arc of the extremal measured from the point $P$ so that for every value of $a$ the point $P$ corresponds on $\mathbb{E}_{a}$ to the value $t=0$.

If we vary $a$, we thus obtain a set of extremals

$$
x=\phi(t, a), \quad y=\psi(t, a),
$$

for which the functions $\phi, \psi$ have the following properties :

1) The functions

$$
\phi, \phi_{t}, \phi_{t t} ; \psi, \psi_{t}, \psi_{t t}
$$

are as functions of $t$ and $a$ of class $C^{\prime}$ in the domain

$$
0 \leqq t \leqq l, \quad A_{1} \leqq a \leqq A_{2},
$$

where $l$ is a sufficiently small positive quantity independent of $a . \ddagger$

2) The functions $\phi, \psi$ satisfy the following initial conditions:

$$
\begin{aligned}
\phi(0, a) & =\tilde{x}(a), & \psi(0, a) & =\tilde{y}(a), \\
\phi_{t}(0, a) & =\tilde{x}^{\prime}(a), & \psi_{t}(0, a) & =\tilde{y}^{\prime}(a) .
\end{aligned}
$$

From (5) we obtain by differentiation

$$
\begin{array}{ll}
\phi_{a}(0, a)=\tilde{x}^{\prime}(a), & \psi_{a}(0, a)=\tilde{y}^{\prime}(a), \\
\phi_{t a}(0, a)=\tilde{x}^{\prime \prime}(a), & \psi_{t a}(0, a)=\tilde{y}^{\prime \prime}(a) .
\end{array}
$$

From these equations we derive for the Jacobian

$$
\Delta(t, a)=\frac{\partial(\phi, \psi)}{\partial(t, a)}
$$

\footnotetext{
* Compare Burss, A nnals of Mathematics, ser. 2, vol. 6 (1905), pp. 49-67.

† Compare KNeser, Lehrbuch der Variationsrechnung, $8 \& 27,29$ and BoLZA, Lectures on the Calculus of Variations, \& 25, $b$ ).

¥Compare the corollary given by BLIss in the article on differential equations just referred to, p. 53, at the end of section 1.
} 
the result :

$$
\Delta(0, a)=0, \quad \Delta_{t}(0, a)=\frac{1}{r}-\frac{1}{\tilde{r}},
$$

if we denote by $1 / r$ and $1 / \tilde{r}$ the curvature at the point $P$ of the curve $\mathbb{E}_{a}$ and of the curve $\widetilde{\mathfrak{E}}$ respectively.

We make the further assumption that

$$
\frac{1}{r}-\frac{1}{\tilde{r}} \neq 0
$$

along $\widetilde{\mathfrak{E}}$, and in order to fix the ideas we suppose * that

$$
\frac{1}{r}-\frac{1}{\tilde{r}}>0 \text {. }
$$

From this additional assumption it follows that two positive quantities $l_{0} \leqq l$ and $m$ can be determined so that

$$
\Delta(t, a) \geqq t m
$$

in the domain

$$
0 \leqq t \leqq l_{0}, \quad A_{1} \leqq a \leqq A_{2} .
$$

For if we define the function $\chi(t, a)$ for the domain (4) by the equations

$$
\chi(t, a)= \begin{cases}\frac{\Delta(t, a)}{t}, & \text { when } t \neq 0, \\ \Delta_{t}(0, a), & \text { when } t=0,\end{cases}
$$

it is easily seen that $\chi(t, a)$ is continuous in the domain (4), and since moreover $\chi(0, a)>0$ in $\left(A_{1} A_{2}\right)$, it follows that a positive quantity $l_{0} \leqq l$ can be assigned such that $\chi(t, a)>0$ in the domain (10). If we denote by $m$ the minimum of $\chi(t, a)$ in the domain (10), we obtain (9).

\section{§ 2. Proof that the set of extremals (3) furnishes a field.}

We now choose two quantities $a_{1}, a_{2}$ so that

$$
A_{1}<a_{1}<a_{2}<A_{2}
$$

and propose to prove that under the assumptions enumerated in $\S 1, a$ positive quantity $k \leqq l_{0}$ can be assigned such that the equations (3) define a one-to-one correspondence between the rectangle

* In order that the curve $\widetilde{夭}$ may furnish a minimum for the integral (1) with respect to onesided variations on the left of $\widetilde{E}$, it is necessary that $1 / r-1 / \widetilde{r} \geqq 0$; compare BoLZA, Lectures, p. 194. 


$$
\mathrm{A}_{\kappa}: \quad 0 \leqq t \leqq \kappa, \quad a_{1} \leqq a \leqq a_{2}
$$

in the $t$, a-plane and its image $\mathbf{s}_{\kappa}$ in the $x, y$-plane.

We suppose that it were not so; that is we suppose that, however small $\kappa$ may be taken, there always exists in $\mathbf{A}_{\kappa}$ at least one pair of distinct points whose images in the $x, y$-plane coincide. Reasoning then exactly as in the proof for the exist-
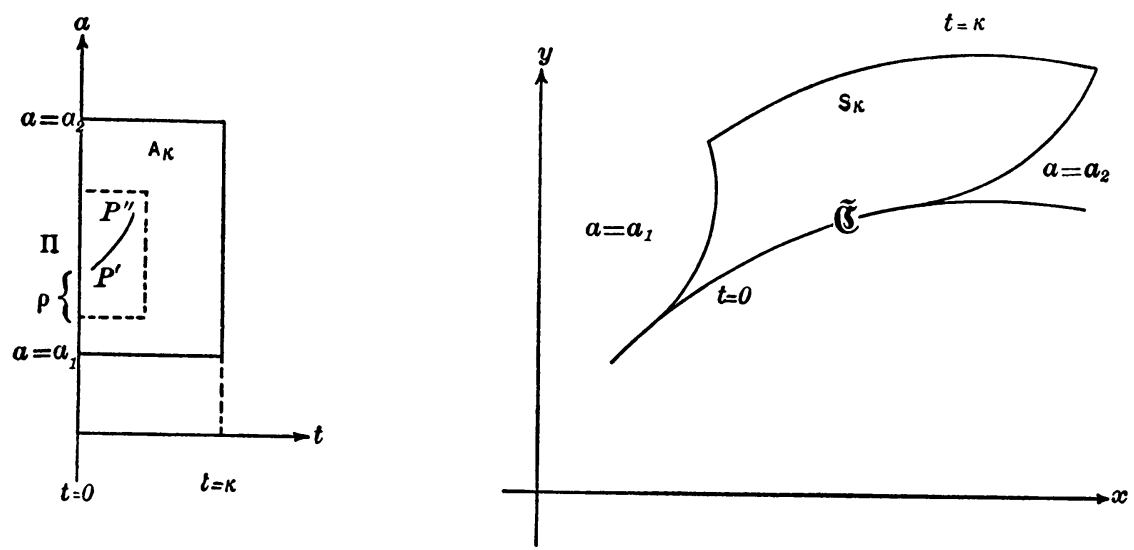

ence of a field which I have given in $\S 34$ of my Lectures for the case where $\Delta(t, a) \neq 0$, we reach the result that under this hypothesis there would exist a point $\Pi(t=0, a=\alpha)$ in the rectangle $\mathbf{A}_{\kappa}$ such that every vicinity of $\Pi$ contains at least one pair of distinct points of $\mathbf{A}_{\kappa}$ whose images in the $x, y$-plane coincide.

We are going to prove that this leads to a contradiction with the inequality (9).

For this purpose we notice that our assumptions concerning the curve $\tilde{\mathbb{E}}$ imply * that $\tilde{x}^{\prime}(\alpha), \tilde{y}^{\prime}(\alpha)$ are not both zero; let $\tilde{x}^{\prime}(\alpha) \neq 0$, or as we may write on account of $(6)$,

$$
\phi_{a}(0, \alpha) \neq 0 .
$$

We may then apply Dinr's theorem on implicit functions to the function $\phi(t, a)$ and the point $t=0, a=\alpha$. From this theorem it follows $\dagger$ that below any

* Compare the definition of "curve of class $C$ " " on p. 116 of my Lectures.

$\dagger$ Choose $d>0$ so that $\phi(t, a)$ is of class $C^{\prime}$ and $\phi_{a}(t, a) \neq 0$ for $|t| \leqq d,|a-a| \leqq d$. Let $A$ be the maximum of $\left|\phi_{t}(t, a)\right|, B$ the minimum of $\left|\phi_{a}(t, a)\right|$ in this domain. Choose $0<d_{0}<d$ and

$$
\sigma<d_{1}-d_{0}, \rho<d_{0}, \frac{\sigma}{2}, \frac{B \sigma}{2 A} .
$$

Compare Peano-Genocchi, Differentialrechnung und Grundzüge der Integralrechnung, pp. 138141. 
preassigned positive quantity $\delta$ two positive quantities $\rho$ and $\sigma$ can be determined having the following properties: If $P^{\prime}\left(t^{\prime}, a^{\prime}\right)$ and $P^{\prime \prime}\left(t^{\prime \prime}, a^{\prime \prime}\right)$ be any two distinct points of the vicinity $(\rho)$ of the point $\Pi(0, \alpha)$ for which

$$
\phi\left(t^{\prime}, a^{\prime}\right)=\phi\left(t^{\prime \prime}, a^{\prime \prime}\right),
$$

then in the first place $t^{\prime \prime} \neq t^{\prime}$ (say $t^{\prime}<t^{\prime \prime}$ ) and in the second place the two points $P^{\prime}, P^{\prime \prime}$ can be joined by a curve representable in the form

such that

$$
a=\mathfrak{a}(t), \quad t^{\prime} \leqq t \leqq t^{\prime \prime},
$$

$$
\phi[t, \mathfrak{a}(t)]=\phi\left(t^{\prime}, a^{\prime}\right) \text { for } t^{\prime} \leqq t \leqq t^{\prime \prime} .
$$

The function $\mathfrak{a}(t)$ is of $C^{\prime}$, and satisfies the inequality

$$
\left.\mid \mathfrak{a}(t)-a^{\prime}\right) \mid<\sigma \text { for } t^{\prime} \leqq t \leqq t^{\prime \prime}
$$

and the initial conditions

$$
\mathfrak{a}\left(t^{\prime}\right)=a^{\prime}, \quad \mathfrak{a}\left(t^{\prime \prime}\right)=a^{\prime \prime} .
$$

Differentiating (12) we obtain

$$
\phi_{t}[t, \mathfrak{a}(t)]+\phi_{a}[t, \mathfrak{a}(t)] \mathfrak{a}^{\prime}(t)=0 .
$$

On the other hand, it follows from the characteristic property of the point $\Pi$ that there exists at least one pair of distinct points $P^{\prime}, P^{\prime \prime}$ in the domain

$$
0 \leqq t<\rho, \quad|a-a|<\rho
$$

for which not only (11) holds but at the same time

$$
\psi\left(t^{\prime}, a^{\prime}\right)=\psi\left(t^{\prime \prime}, a^{\prime \prime}\right)
$$

For such a pair of points the function $\psi[t, \mathfrak{a}(t)]$ is of class $C^{\prime}$ in $\left(t^{\prime} t^{\prime \prime}\right)$ and takes, according to (13) and (15), the same value for $t=t^{\prime}$ and $t=t^{\prime \prime}$. Hence its derivative must vanish at least for one value $t=\tau$ between $t^{\prime}$ and $t^{\prime \prime}$ :

$$
\psi_{t}(\tau, \mathfrak{a}(\tau)]+\psi_{a}[\tau, \mathfrak{a}(\tau)] \mathfrak{a}^{\prime}(\tau)=0 .
$$

Combining this equation with the equation derived from (14) by putting $t=\tau$, we obtain the result:

$$
\Delta[\tau, \mathfrak{a}(\tau)]=0 .
$$

But if we take $\rho$ and $\sigma$ sufficiently small, the point $t=\tau, a=\mathfrak{a}(\tau)$ lies in the domain (10); moreover, $\tau$ is positive since $0 \leqq t^{\prime}<\tau<t^{\prime \prime}$. Hence we have indeed reached a contradiction with the inequality (9), and therefore the statement enunciated at the beginning of this section is proved. 
The image $\mathfrak{R}$ of the boundary of the rectangle $A_{\kappa}$ is a continuous closed curve without multiple points. According to a theorem due to Schönflies, ${ }^{*}$ the point-set $\mathbf{S}_{\kappa}$ is therefore identical with the interior of $\mathfrak{R}$ together with the curve $\mathfrak{R}$ itself.

The University of Chicago, February 27, 1907.

*Göttinger Nachrichten, 1899, p. 282 ; compare also Osaood, ibid., 1900, p. 94 ; and Bernstein, ibid., 1900, p. 98. 